\title{
Heterogeneous Memristive Crossbar for In-Memory Computing
}

\author{
Georgios Papandroulidakis, Ioannis \\ Vourkas, and Georgios Ch. Sirakoulis \\ Dept. of Electrical and Computer Eng. \\ Democritus University of Thrace (DUTh) \\ Xanthi, Greece \\ \{georpapa17, ivourkas, \\ gsirak\}@ee.duth.gr
}

\author{
Antonio Rubio \\ Dept. of Electronic Engineering \\ Polytechnic University of Catalonia (UPC) \\ Barcelona Tech, Barcelona, Spain \\ antonio.rubio@upc.edu
}

\section{MEMRISYS2015 Extended Abstract}

The memristor represents one of today's latest technological achievements in circuits and systems, demonstrating advantageous characteristics which open new pathways for the exploration of advanced computing paradigms and beyond-Von Neumann architectures [1], [2], [3]. Most importantly, it provides with an unconventional computation framework which combines information processing and storage in the memory itself; i.e. the major distinction from the present day's computing technology [4]. Moreover, memristor-based crossbar nanoarchitectures are highly considered as candidate structures for future memory and logic applications, where the same physical devices are used either as storage elements or logic gates [5], [6], [7], [8], [9].

In this work we propose the design of a novel crossbar geometry, which is heterogeneous in terms of its cross-point devices, allowing for the realization of true in-memory digital logic computations. More specifically, it is a combination of two stacked crossbar arrays with a shared intermediate nanowire layer. The variety of available cross-points types allows the execution of parallel memristive logic computations, where the logic state variable is voltage [10]. Moreover, the utilization of insulating patterns in the crossbar arrays, at the expense of a small area-overhead, permits the simultaneous parallel read/write memory operation of two memory words [8]. Memory/logic operation is determined through control signals driven from the peripheral CMOS-based driving circuitry, which also comprises row/column decoders, tri-state drivers, and summing/sense amplifiers to allow for the proper programming/reading of the memristive cross-points. A simulation-based validation of read/write memory operations as well as of parallel memristive logic computations (half adder, sum of products, etc.) is performed using the PSPICE simulation environment and a device model of a threshold-type, voltage-controlled, bipolar memristor. The outcome of this work includes combined dense storage/computing nanoarchitectures, based on memristors, to be used in future electronic computing systems.

\section{Tentative List of References}

[1] L. O. Chua, "If it's pinched it's a memristor," Semicond. Sci. Technol., vol. 29, no. 10 (104001), 2014

[2] "International Technology Roadmap for Semiconductors (ITRS)," 2013. [Online]. Available: http://www.itrs.net/. [Accessed June 2014] 
[3] W. Zhao, D. Querlioz, J. O. Klein, D. Chabi and C. Chappert, "Nanodevice-based Novel Computing Paradigms and the Neuromorphic Approach," 2012 IEEE Int. Symp. Circuits and Syst. (ISCAS), Seoul, South Korea, May 20-23, pp. 2509 - 2512

[4] M. Di Ventra and Y. V. Pershin, "The parallel approach," Nature Physics, vol. 9, pp. 200-202, April 2013

[5] E. Linn, R. Rosezin, S. Tappertzhofen, U. Bottger and R. Waser, "Beyond von Neumann-logic operations in passive crossbar arrays alongside memory operations," Nanotechnology, vol. 23, no. 30 (305205), 2012

[6] S. Paul, and S. Bhunia, "A scalable memory-based reconfigurable computing framework for nanoscale crossbar," IEEE Trans. Nanotechnol., vol. 11, no. 3, pp. 451462,2012

[7] O. Kavehei, S. Al-Sarawi, K.-R. Cho, K. Eshraghian, and D. Abbott, "An Analytical Approach for Memristive Nanoarchitectures," IEEE Trans. Nanotechnol., vol. 11, no. 2, pp. $374-385,2012$

[8] I. Vourkas, D. Stathis, G. Ch. Sirakoulis, and S. Hamdioui, "Alternative Architectures towards Reliable Memristive Crossbar Memories," IEEE Trans. VLSI Syst. (2015), in press doi: 10.1109/TVLSI.2015.2388587

[9] P. Pouyan, E. Amat, and A. Rubio, "Reliability challenges in design of memristive memories," 2014 European Workshop on CMOS Variability (VARI), Palma de Mallorca, Spain, Sept. 29-Oct. 1, pp. 1-6

[10] G. Papandroulidakis, I. Vourkas, N. Vasileiadis, and G. Ch. Sirakoulis, "Boolean Logic Operations and Computing Circuits Based on Memristors," IEEE Trans. Circuits Syst. II, Expr. Briefs, vol. 61, no. 12, pp. 972-976, 2014 